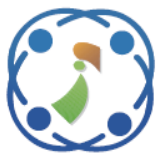

\title{
Detecting Diabetic Retinopathy from Retinal Images Using CUDA Deep Neural Network
}

\author{
Ricky Parmar ${ }^{1 *} \quad$ Ramanathan Lakshmanan $^{1}$ \\ ${ }^{1}$ School of Computer Science and Engineering, Vellore Institute of Technology, Tamil Nadu India \\ * Corresponding author's Email: parmar_ricky@yahoo.in
}

\begin{abstract}
A microvascular impediment, Diabetic Retinopathy, is observed due to long term diabetes and is one of the significant reason for visual impedance because of variations in veins of the retina. Significant loss of vision due to Diabetic Retinopathy can be profoundly prevented with proper screening and medication at a more earlier stage. The evaluating procedure comprises of perceiving fine points of interest, for example, microaneurysms, to some greater elements, for example, exudates, and some of the time their position in respect to each other on pictures of the eye. Our task here is to predict the class for each of the images. We classify our images into five categories We are using Convolutional Neural Networks for our prediction model and we train our model on GPU. GPU-accelerated library of primitives aimed at Deep Neural Networks, NVIDIA CUDA Deep Neural Network (cuDNN) is used in our model. Our model has around $85 \%$ of accuracy when tested on 53576 number of retinal images. Our solution is elegant and automated, saving a lot of time and manual efforts.
\end{abstract}

Keywords: Diabetic retinopathy, Diabetes, Convolutional neural networks, CUDA, Deep neural network, Deep learning.

\section{Introduction}

In this populace of working age, Diabetic Retinopathy (DR) is considered as the main source of visual deficiency. It has been assessed that this has affected more than 93M (million) individuals living in this developed world. Number of individuals in year 2013, all over the globe, suffering from diabetes, has gotten up to $382 \mathrm{M}$ and by the year 2035 , it has been predicted to reach around 592M [1]. DR in its early phase can be described by its qualities, for example, inconsistencies and defectiveness of veins, however if this conditions become serious, this may lead to blindness. Recognizing DR, as of now is a manual and tedious process which requires analysis and assessment of computerized colour fundus retinal images by a qualified clinician. Diabetic Retinopathy can be identified by a qualified clinician by observing association of lesions with vascular anomalies which are caused by the ailment. Despite of it being an effective approach, request for resource is high with this methodology. There frequently is a circumstance of resource non-availability, such as medical expertise and equipment, in region where the percentage of diabetes is high in native populace. Such places are in most need of Diabetic Retinopathy detection. With time, number of individuals with diabetes keeps on growing, and with this growing number of patient's, infrastructure to avoid blindness

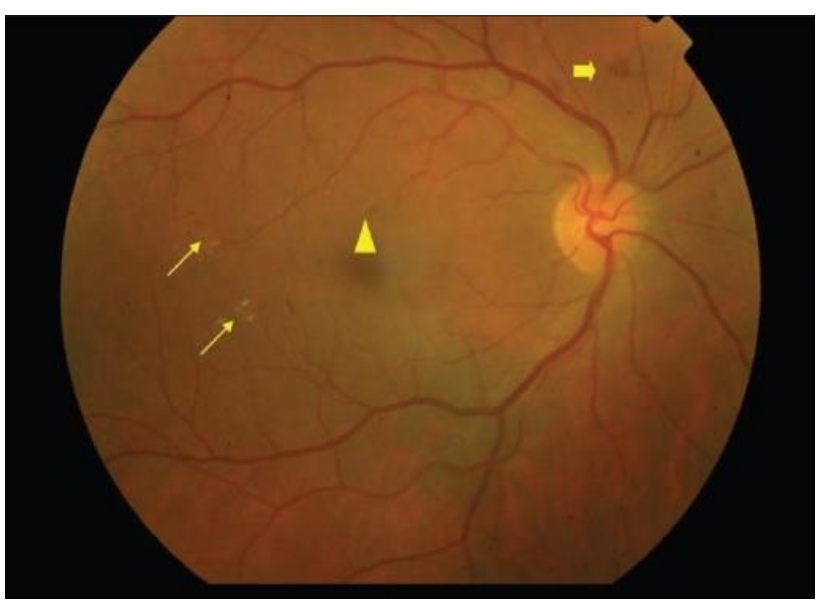

Figure.1 Non-proliferative diabetic retinopathy 
caused by Diabetic Retinopathy will be even more inadequate. Being a non-painful disease, visual defect is not observed till the late stages, when it becomes very difficult to treat. Treatment at this stage is mostly ineffective. To make sure that patient is treated on time by proper diagnosis of Diabetic Retinopathy at an initial stage, multiple screening of the patient must be done at that stage [2,3], where retinal picture analysis is a noteworthy part. As all the essential resources for this process are not available in all the region around the world, the need for automated screening of Diabetic Retinopathy is recognized. Our evaluating procedure includes microaneurysms which are minor details as well as exudates which is a major feature. There are many occasions where the relative position of microaneurysms to exudates is also considered as a feature.

In Fig. 1, Hard exudates are indicated by Thin Arrows, internal retinal haemorrhage is depicted by thick arrow, whereas microaneurysms are shown by thick arrow. Per [4,5], improvement in computerized classifications to recognize Diabetic Retinopathy has become of an incredible significance for the research communal. Harangi et al. [6] have recognized the locales containing exudates in retinal pictures by utilizing greyscale morphology and afterward dynamic form based technique was utilized to extricate the exact fringes of the hopefuls.

Research works such as [7] have called attention to that the profound structures, for example, multilayer neural systems, demonstrated better execution when the customary backpropagation calculation was gone before by an unsupervised pretraining. This brought back the enthusiasm of the scholarly club into multilayer neural systems once more, and profound learning techniques [8] have since surpassed expectations in being the top entertainer in numerous applications, for example, question identification and acknowledgment, speaker identification [9], content mining [7] and handwriting recognition [10]. Although a solid numerical thinking of the achievement behind these systems is yet hazy, a few natural reasons have been explained in [11] and [12]. Walter [13] has proposed another system, in which certain chose elements, for example, microaneurysms alone are improved. Cree et al. [14] have connected a picture reclamation method for pictures of exceptionally low quality. Image restoration technique where images are considered as trial functions of stochastic procedure was used by peli [15].

Using intensity properties for dynamic clustering, Hsu et al. [16-17] could find normal and abnormal areas of images. Exact contours were found using morphological reconstruction and high grey-level variation and in this manner exudates were detected by [17]. A new method using fuzzy c-means to detect abnormal region was proposed by zang [18]. Another method for exudate detection with statistical classification by applying brightness adjustment by proposed by Wang et al [19].

In [20], authors have used a dataset of merely 60 images and used SVM algorithm for classification and have only done dual classification. While in [21], they only focus on exudates and use basic algorithm such as random forest. So, diabetic retinopathy caused by microaneurysms are not detected. Whereas [22], Only focuses on microaneurysms for the early detection of diabetic retinopathy. Also, all [20-22] classify images into two classes. While in compare to [20-22] our model uses latest state-of-art algorithm such as Convolution Neural Network, and takes advantage of the very powerful GPU by using NVIDIA CUDA Deep Neural Network (cuDNN). Also, we use a huge dataset of 53576 number of images for testing our model (validation) and train on 35100 number of images. Data used is around 85 GB in size. Our model focuses on both exudates and microaneurysms for early detection of diabetic retinopathy. For microaneurysms detection we use digital artifact effect explained in section 2. Unlike any other previous methods which does only dual classification, our model does multiclass classification and classify images into five categories (i.e. Normal, Mild NPDR, Moderate NPDR, Severe NPDR, PDR) with weighted mean sensitivity of 0.85 and specificity of 0.81 . Also, to predict the class of 53576 number of pictures, it took only 21 mins for our algorithm (dimension reduced to $512 \times 512$ ) and for huge size image (dimension 4752x3186) it takes only 4 hours to predict the class of all 53576 images.

This paper is organized as follows: Section 2 provides an overview of our model. Such as, preprocessing, describing architecture of the convolutional neural network in our model, techniques we used in our model. In Section 3 we discuss results of our model as well as other various analysis such as accuracy of the model and statistics such as specificity and sensitivity of the model. Whereas, Section 4 of the paper has conclusion

\section{Method}

We use around 35100 pictures (of retina) as training set in our examination. Where each image is marked by patient id and whether it's a left eye image or right (e.g. 234_left.jpg). Images are per eye, not per patient. Therefore, there are two images that belong to each patient. These pictures are distributed 
Table 1. Class distribution

\begin{tabular}{cccc}
\hline Name & Class\# & \#Images & \% Dist. \\
\hline Normal & 0 & 25810 & $73.48 \%$ \\
\hline $\begin{array}{c}\text { Mild } \\
\text { NPDR }\end{array}$ & 1 & 2443 & $6.96 \%$ \\
\hline $\begin{array}{c}\text { Moderate } \\
\text { NPDR }\end{array}$ & 2 & 5292 & $15.07 \%$ \\
\hline $\begin{array}{c}\text { Severe } \\
\text { NPDR }\end{array}$ & 3 & 873 & $2.48 \%$ \\
\hline PDR & 4 & 708 & $2.01 \%$ \\
\hline
\end{tabular}

into five classes which one can state are equally unhinged. Following is the image distribution in training set per class.

We have about 53576 number of pictures as a test case and we classify these images into above mentioned five classes. Data for retinal images is provided by EyePACS. The most predominant soonest indication of diabetic macular oedema relates to the nearness of exudates inside the macular district. Henceforth, the assignment of identifying exudates by utilizing Computer based examination assumes an indispensable part in the conclusion of Diabetic Retinopathy [23]. These exudates have high-dark level variety from its encompassing foundation furthermore, consequently they are much obvious as splendid examples in shading fundus pictures [24]. In our proposed work, we identify these exudates, microaneurysms and internal retinal haemorrhage from our retinal images.

\subsection{Pre-processing}

Our initial phase of Pre-processing these images would be to, downscale them, as original images are quite huge in their dimension i.e. around $4752 \times 3186$ pixel approximately. We decide to downscale these images about 5 times of their actual size which would be around $512 \times 512$ pixel, without interpolation. Below is example before initial pre-processing of image. It is evident from the Fig. 2 that each image varies in size and there is black border around it, so we intend to remove that in this phase. Fig. 3 Depict same image after processing it through first phase. Task of enhancing these images computationally was made further easier with these new set of images. Next phase of pre-processing will now involve various enhancement of images such as resizing, cropping (with certain probability), zooming, flipping images, rotating, colour balance, contrast, brightness etc.

While training, arbitrary specimens are selected from the training set and are then transformed beforehand, then are queued for input to the network. The enhancements were done dynamically during training on images by spawning a thread whenever needed from the CPU, it was taken care of that no delay is observed, in waiting, for samples to process. Resizing of the images had to be taken care of before any other enhancements and before resizing, images were cropped. If it were not done, the computation would be too intensive. This resizing altered the original aspect ratio of the image by using normal bilinear rescaling. To normalize our input, we subtract total mean from it and after that we divide it with standard deviation, which is estimated on few hundred of samples before training, this normalization is done during training.

\subsection{Architecture}

In our input, we have two retinal images for every patient, which is each per eye (left and right).

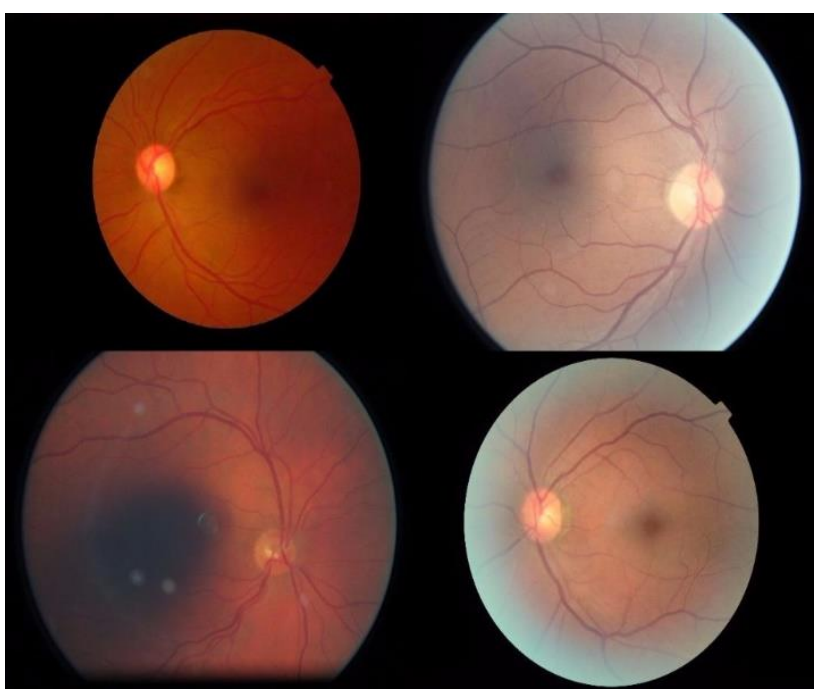

Figure.2 Before initial pre-processing

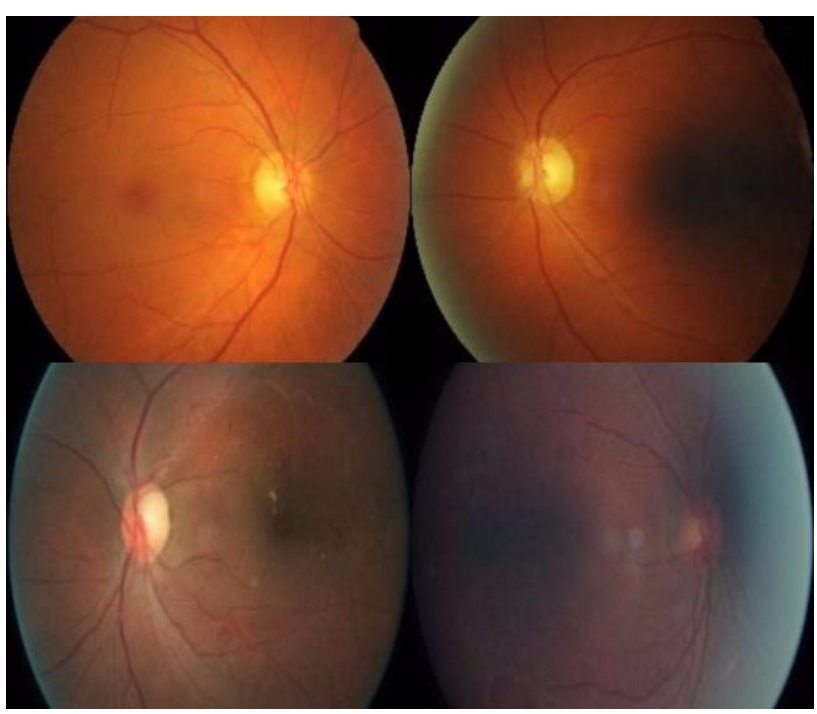

Figure.3 After initial pre-processing 
We use both the images to classify individual image by coalescing them before the last two dense layers, out of the two-dense layer at the end one of them is SoftMax layer. Below is the architecture for $512 \times 512$ input.

Table 2. Architecture

\begin{tabular}{|c|c|c|c|c|c|}
\hline Layer \# & $\begin{array}{l}\text { Layer } \\
\text { Name }\end{array}$ & Batch Size & Channels & Width & Height \\
\hline 0 & Input & 64 & 3 & 512 & 512 \\
\hline 1 & Convnet & 64 & 32 & 256 & 256 \\
\hline 2 & $\begin{array}{c}\text { Max } \\
\text { Pooling }\end{array}$ & 64 & 32 & 127 & 127 \\
\hline 3 & Convnet & 64 & 32 & 127 & 127 \\
\hline 4 & Convnet & 64 & 32 & 127 & 127 \\
\hline 5 & $\begin{array}{c}\text { Max } \\
\text { Pooling }\end{array}$ & 64 & 32 & 63 & 63 \\
\hline 6 & Convnet & 64 & 64 & 63 & 63 \\
\hline 7 & Convnet & 64 & 64 & 63 & 63 \\
\hline 8 & $\begin{array}{c}\text { Max } \\
\text { Pooling }\end{array}$ & 64 & 64 & 31 & 31 \\
\hline 9 & Convnet & 64 & 128 & 31 & 31 \\
\hline 10 & Convnet & 64 & 128 & 31 & 31 \\
\hline 11 & Convnet & 64 & 128 & 31 & 31 \\
\hline 12 & Convnet & 64 & 128 & 31 & 31 \\
\hline 13 & $\begin{array}{c}\text { Max } \\
\text { Pooling }\end{array}$ & 64 & 128 & 15 & 15 \\
\hline 14 & Convnet & 64 & 256 & 15 & 15 \\
\hline 15 & Convnet & 64 & 256 & 15 & 15 \\
\hline 16 & Convnet & 64 & 256 & 15 & 15 \\
\hline 17 & Convnet & 64 & 256 & 15 & 15 \\
\hline 18 & $\begin{array}{c}\text { Max } \\
\text { Pooling }\end{array}$ & 64 & 256 & 7 & 7 \\
\hline 19 & Drop-out & 64 & 256 & 7 & 7 \\
\hline 20 & $\begin{array}{l}\text { Dense } \\
\text { Layer }\end{array}$ & 64 & 1024 & & \\
\hline 21 & $\begin{array}{c}\text { Feature } \\
\text { Pool } \\
\text { Layer }\end{array}$ & 64 & 512 & & \\
\hline 22 & $\begin{array}{l}\text { Concat } \\
\text { Layer }\end{array}$ & 64 & 514 & & \\
\hline 23 & $\begin{array}{l}\text { Reshape } \\
\text { Layer }\end{array}$ & 32 & 1028 & & \\
\hline 24 & Drop-out & 32 & 1028 & & \\
\hline 25 & $\begin{array}{l}\text { Dense } \\
\text { Layer }\end{array}$ & 32 & 1024 & & \\
\hline 26 & $\begin{array}{c}\text { Feature } \\
\text { Pool } \\
\text { Layer }\end{array}$ & 32 & 512 & & \\
\hline 27 & Drop-out & 32 & 512 & & \\
\hline 28 & $\begin{array}{l}\text { Dense } \\
\text { Layer }\end{array}$ & 32 & 10 & & \\
\hline 29 & $\begin{array}{l}\text { Reshape } \\
\text { Layer }\end{array}$ & 64 & 5 & & \\
\hline 30 & $\begin{array}{c}\text { Apply } \\
\text { Non- } \\
\text { linearity }\end{array}$ & 64 & 5 & & \\
\hline 31 & Softmax & 64 & 5 & & \\
\hline
\end{tabular}

First Layer in our neural net (i.e. Layer 0) is input layer, which is mentioned as 'Input' in Table 2, our input to the neural net is [3x512x512], which is raw pixel values of the retinal image which has width 512 and height 512, where there are 3 colour channel i.e. RGB.

Next Layer will be convnet (i.e. Layer 1), we perform a $2 \mathrm{D}$ convolution on our input data with 32 filters, so that makes the layer as [32x256x256], and we also add bias and elementwise nonlinearity. This layer will process the outputs of the locally connected neurons in input. After this there is max-pool layer (i.e. Layer 2), where we perform a down-sampling operation on the previous layer, which results in volume such as [32x127x127]. Now, till 'Layer 18' in our neural net we make combination of convolution and max pooling layer for optimization of our model.

Now we have a drop-out layer (i.e. Layer 19,24 $\& 27$ ), we use this layer randomly between some layer to reduce the overfitting. This layer acts as a regularizer which randomly sets input to 0 which in turn takes care of overfitting. Next, we have Dense Layer (i.e. Layer 20), these are fully connected layers of our neural network. After Dense layer, we have Feature Pooling Layer, we use this layer for 2D Maxout, it is simply a layer where the activation function is max of the inputs. Here the drop-out only occurs in the linear part of our network, so we use maxout to approximate our activation function. It can also pool across a given axis of input. But, we use it to implement maxout.

After Feature pool layer, we use Concat Layer, which as the name suggests concatenates multiple inputs along the axis we specify. This layer is used to merge two images (i.e. 2 eyes per patient, so we can detect patterns created by digital artifact). Following Concat Layer is Reshape Layer, which does the reshaping of its input tensor to another tensor of the similar overall numeral of elements. Then again, we optimize our neural network with different combination of layer. Next layer (layer 30) which is Non-Linearity Layer, which simply applies nonlinearity. Our last layer is softmax layer (Layer 31) which is fully connected layer which computes class scores, resulting in channel of 5, i.e. 5 classes in which we classify our images.

Most crucial effect on performance of the model was by using leaky rectify units with comparatively higher leakiness. Initially, alpha value was kept to be 0.3 , but after few run it was changed to 0.5 to get higher accuracy. Accuracy will be even increased more if we do not downscale the images to five times, which we did in the initial pre-processing stage. Even downscaling the image to two times of the original 
will have a lot of effect on accuracy. But, the hardware used was not powerful enough to carry out that intensive computation, so images with downscaled up to five times were used. The overfitting of rather subsequent uniform distribution of smaller classes was done. Reshape layer in our architecture is the place where images for each patient are merged to one in first reshape layer, while in second they are split to two again.

\subsection{Digital artifacts}

When we take a glimpse at two pictures of certain patient, keeping them beside each other, there is something noticeable (Digital artifact) which can be seen, which absolutely can't be seen when looking at a single picture. Fig. 4 shows an example of digital artifact, Dark spots and stripes on the external right side and small dark dabs on the external left side. They resemble microaneurysms and in some cases, they are, while in others it's just tiny black dots, so to figure out when it is microaneurysms, we need both the images of patient. So, it is very unlikely that the model can distinguish with just single image and catch microaneurysms. A lot of spatial information is gotten rid of when layers are fully connected. But, merging two images (with dense representation) means it has very high level of representation. Also, enhancement on each image were done separately, independent of patient. Because paired enhancement will not lead to any improvement.

What we do here is take output ' $x$ ' and ' $y$ ' from the output of some layer (two outputs being for each eye of the patient) and exchanged them with 'xy' and ' $\mathrm{yx}$ ' and stacked them on the channel. Which brings about nets having entry to lower representation and not losing any input space. Instead, if we had merged the outputs of the first convolution layer for each of two eyes then, theoretically our model would be able to recognize similar patterns for each eye of patient. However, input space was reduced way too much with this approach. Thus, the before mentioned method (stacking) was carried out. Because merging had to be done in initial few layers of the network.

\subsection{Pseudo classification}

This strategy utilizes the predictions from different ensembles of model on the test set to help manage or normalize new model. While training some set of images from the test set where added to the batches in training set, roughly around $25 \%$ of the batch contained pictures from test set which were predicted with SoftMax from ensemble.

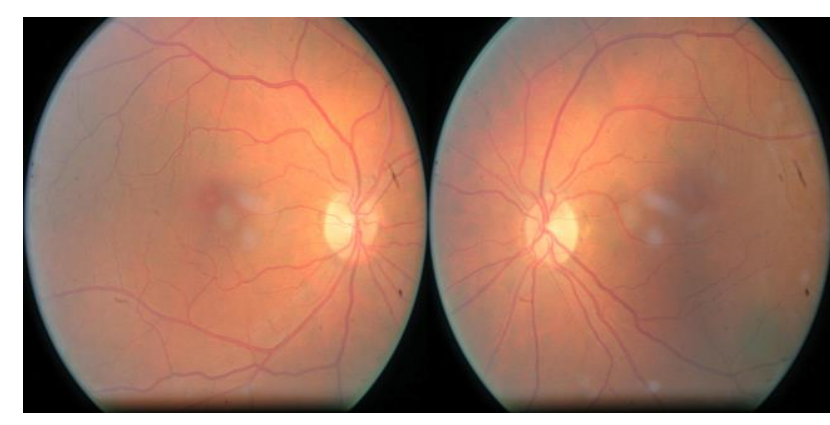

Figure.4 Digital artifact

\section{Results}

Using pseudo classification helped in pushing the model initially to accuracy of 0.83 .

\subsection{Exponential power distribution}

Few things that emerged were how different models varied on the different validation sets. Discrete metric is the reason behind this. While evaluating our model on quadratic kappa metric, its calculated by,

$$
\mathrm{K}=1-\frac{\sum_{i, j} W_{i, j} c_{i, j}}{\sum_{i, j} W_{i, j} E r_{i, j}}
$$

In Eq. (1), Er is $m$ by m matrix of expected rating, $\mathrm{W}$ is $\mathrm{m}$ by $\mathrm{m}$ matrix of weights, whereas $\mathrm{C}_{\mathrm{i}, \mathrm{j}}$ corresponds to images which received rating ' $i$ ' by ' $x$ ' and rating ' $j$ ' by ' $y$ '. Loss from quadratic kappa is calculated by two important metrics let's call it 'nomi' and 'denomi'. $1-\sum$ (nomi) $/ \sum$ (denomi) is how kappa evaluation score is given. Therefore, we would like to minimize 'nomi' as much as possible and look to maximize 'denomi' as much as possible. 'denomi' being stable encapsulates distributions of forecasts and the targets. Whereas we get most of insights of our model from 'nomi'. Following is the look at 'nomi' and 'denomi' for model with 0.83 score on kappa metric. Whereby the frameworks are standardized and demonstrate the rate of the aggregate total situated in that position. The mistakes of foreseeing class practically rule the error ' 0 ' while it was truly class ' 2 ' (which means moderate NPR is predicted as normal eye). The issue with this, and overall, was that the ground truth didn't know by any stretch of the imagination. In Fig. 5 and Fig. 6, X-axis represents predicted class and $\mathrm{Y}$-axis indicates actual class. Both the figures indicate the distribution of error, where Fig. 6 is the normalized version

\subsection{Ensemble}

Ensembling multiple models brought about a decent change by utilizing the log probabilities for 
every class by averaging them, then again bringing them back to their typical original probabilistic state of $[0,1]$ and utilizing the positioning unraveling technique from one of the past sections to relegate names to the pictures. Best kappa score generated by our model would be of +0.84 .

\subsection{Accuracy and stats}

Our model has overall accuracy of 0.8496 (85\% approx.) and following are the statistics per class. Fig. 7 shows the graph for the confusion matrix, where $\mathrm{x}$ axis represents the predicted class and $\mathrm{y}$-axis denotes actual class. (where 0,1,2,3 and 4 are class names). We trained our model on 35100 number of retinal images, where images were pre-processed and the dimension of the image was reduced to $512 \times 512$. This model was trained on GPU with 4GB of memory, we used NVIDIA GPU, namely GeForce GTX 980, which has 2048 number of CUDA cores, it takes about less than one day of time for training the model on this device. If we train the model on low config GPU for e.g. GTX 960 or 970, model takes 3-4 days to train. One can simply use parallel or multiple graphics card to speed up the process. Then It may take even less than 24 hours of time.

We ran our model on about 53,576 number of images. Table 4 describes confusion matrix for our model. Table 3 describes the statistics of the model, like specificity and sensitivity for each class. Model has weighted mean sensitivity of 0.85 and specificity of 0.81 .

Once we trained the model we can store the model dumps into a pickle file, so we can make quick predictions on our image using those dumps. To on all the images in test set i.e. 53576 images.

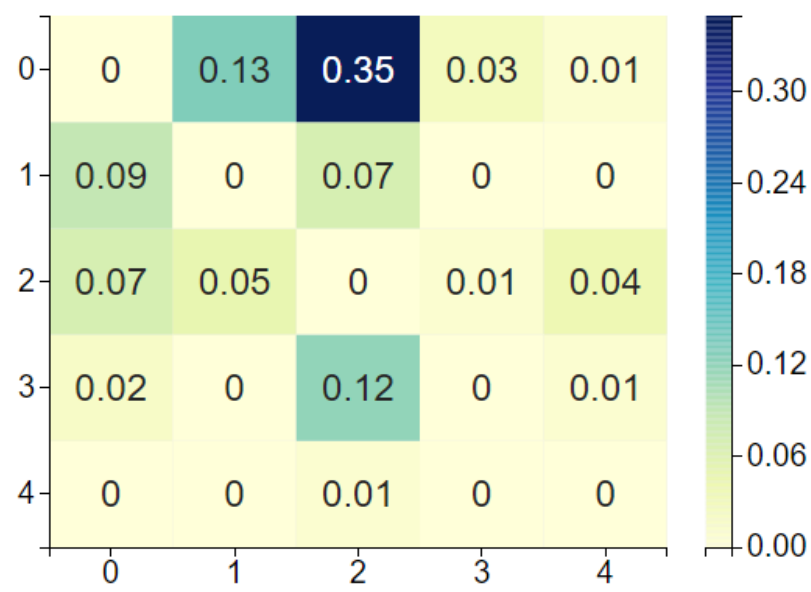

Figure.5 Normalized 'nomi', for $(a, b)$, for actual label b, predicted a

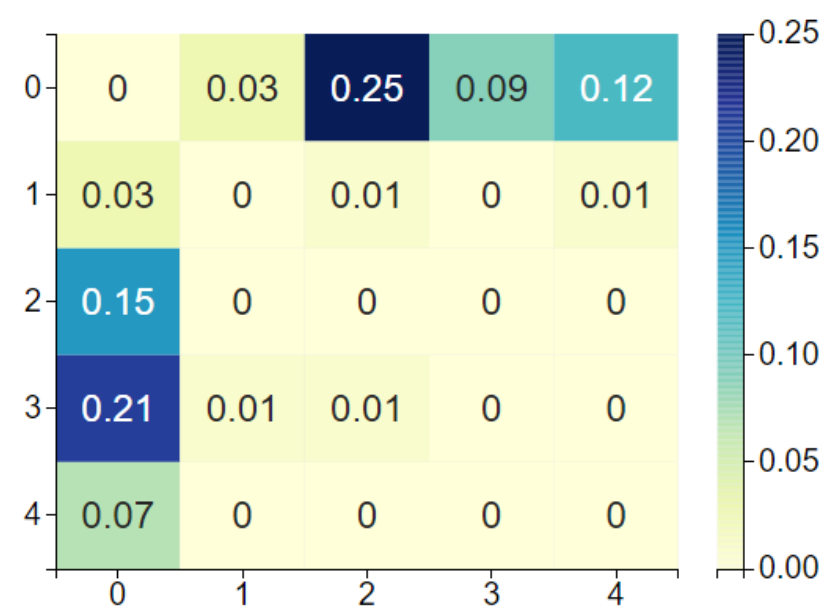

Figure.6 Normalized 'denomi', for $(\mathrm{a}, \mathrm{b})$, for actual label $\mathrm{b}$, predicted a

Table 3. Classification Stats

\begin{tabular}{cccccc}
\hline Classes & 0 & 1 & 2 & 3 & 4 \\
\hline Sensitivity & 0.95 & 0.27 & 0.58 & 0.69 & 0.93 \\
\hline Specificity & 0.91 & 0.97 & 0.96 & 0.97 & 0.99 \\
\hline Accuracy & 0.89 & 0.92 & 0.90 & 0.97 & 0.99 \\
\hline F1 Score & 0.93 & 0.333 & 0.641 & 0.545 & 0.882 \\
\hline
\end{tabular}

Table 4. Confusion matrix

\begin{tabular}{ccccccc}
\hline Predicted & 0 & 1 & 2 & 3 & 4 & All \\
\hline Actual & & & & & & \\
\hline 0 & 37608 & 1009 & 879 & 11 & 26 & 39533 \\
\hline 1 & 2040 & 706 & 990 & 20 & 6 & 3762 \\
\hline 2 & 1372 & 449 & 3900 & 1928 & 212 & 7861 \\
\hline 3 & 31 & 6 & 204 & 806 & 167 & 1214 \\
\hline 4 & 72 & 5 & 160 & 347 & 622 & 1206 \\
\hline All & 41123 & 2175 & 6133 & 3112 & 1033 & $\mathbf{5 3 5 7 6}$ \\
\hline
\end{tabular}

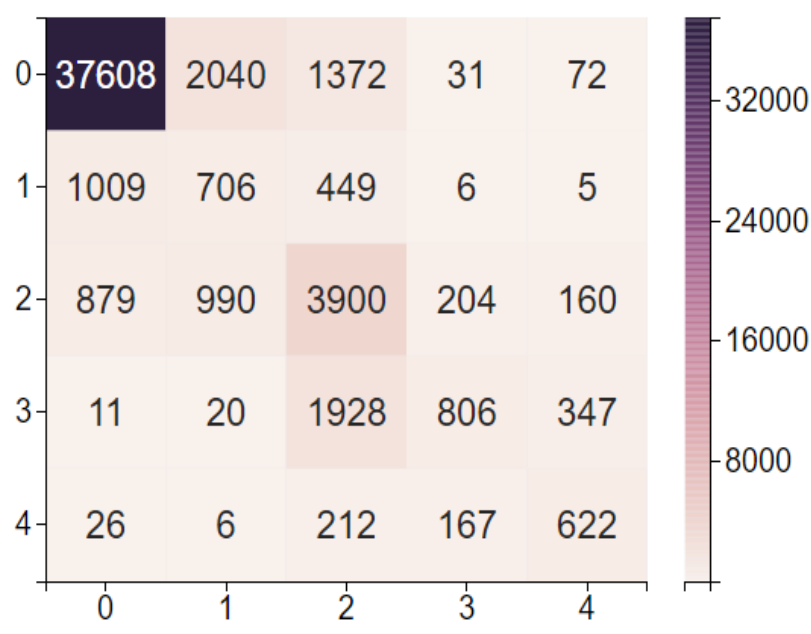

Figure.7 Graph for confusion matrix

For testing, images are loaded into GPU in batches of 256. So, GPU with more memory and speed means faster predictions and training. Performance of the proposed method is measured by specificity and sensitivity of the model. Table 5 shows comparison with various methods for 
specificity and sensitivity. All the present method does the classification of the images in two class (binary classification), our proposed method classifies image into five classes (multiclass classification), which gives more clarification on how alert patient should be so that it can be detected at earlier stage whereas other methods fail to do that. Hence, in comparisons with other method, we considered only class 0 and class 4 (i.e. normal and PDR). Our method took 21 mins to classify 53576 number of images. Which means 42 images per second when images are at $512 \times 512$. When we use high dimension images (i.e. $4752 \times 3186$ ), which takes 4 hours to classify, still classifying 4 images per second. Hence, our model is more accurate and much faster than any other previous method. Also, our model focuses on both exudates as well as microaneurysms which [20-22] and [25] fail to do.

In Table 5 we show comparison of our proposed model with the current existing model. We have compared with following methods, SVM and voting classifier in [20], Random forest classifier in [21] and watershed transform technique in [25]. Fig. 8 shows the bar chart for each sensitivity and specificity against each method. Where y-axis is sensitivity in graph (a) and specificity in graph (b), whereas $x$-axis show various methods against which we compared our proposed method. Bar chart is graphical representation of Table 5.

\section{Conclusion and future work}

An effective technique for the identification and division of the exudates as well as microaneurysms from the retinal pictures, which plays a critical part in the finding of Diabetic Retinopathy has been exhibited in this paper. In paper, we have examined and exhibited a novel method which consequently distinguishes the exudates and microaneurysms from the DR patient's retinal pictures.

Table 5. Comparisons with various methods

\begin{tabular}{ccc}
\hline Method & Sensitivity & Specificity \\
\hline $\begin{array}{c}\text { SVM and Voting } \\
\text { Classifier, [20] }\end{array}$ & 0.86 & 0.94 \\
\hline $\begin{array}{c}\text { Random Forest } \\
\text { classification, [21] }\end{array}$ & 0.83 & 0.76 \\
\hline $\begin{array}{c}\text { Watershed } \\
\text { Transform } \\
\text { Technique, [25] }\end{array}$ & 0.85 & 0.83 \\
\hline Proposed Method & 0.94 & \\
\hline
\end{tabular}

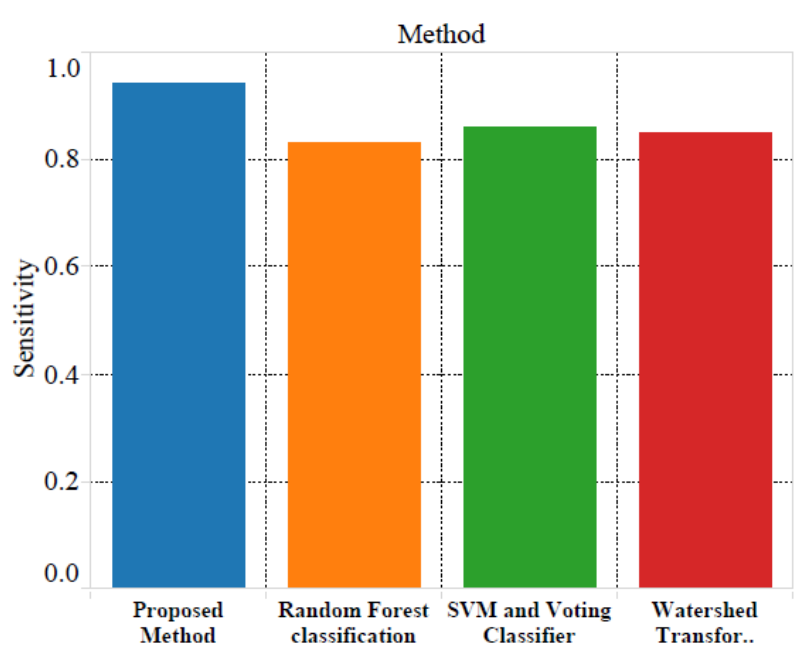

(a)

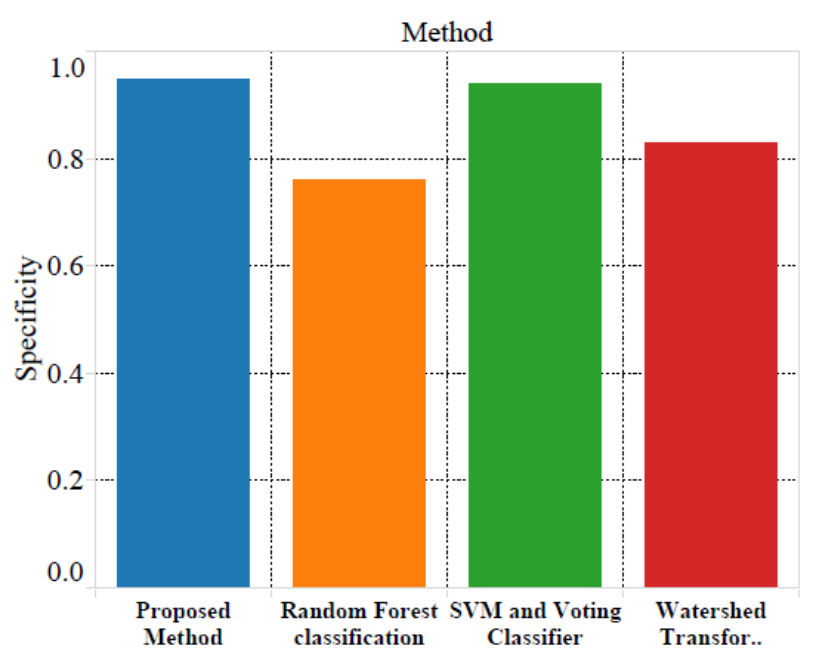

(b)

Figure.8 Comparison against each method: (a) sensitivity comparison and (b) specificity comparison

Shading data was utilized as a part of the preprocessing phase of our calculation and in this way better execution was accomplished in the picture division arrange. It is thought to be an essential highlight to recognize distinctive sores introduce in the shading fundus pictures. Training stage in this work demands intense computational work to identify microaneurysms and exudates from retinal images, using Convolutional Neural Network and powerful GPU (like NVIDIA GTX 980). Training takes around 80 hours of time when it is run on this hardware. There are many other methods to detect diabetic retinopathy from retinal images but most of those approaches are manual where we construct several number of filters and use pipelines to extract features from images. But, convnets can do all that on its own, in a way this solution is more accurate and elegant than other previous solutions. Huge variance from extremely small classes, noisy labels and 
discrete metrics which heavily affects misclassification were big issues during the process. Enhancing learning on the extraordinary kappa metric appeared to be of a great deal more imperative than improving the design.

Our approach achieved around $85 \%$ of accuracy, with good mean sensitivity as well as specificity, and it was tested on very huge amount of data. i.e. 53576 number of high resolution images. This screening framework is reasonably very less demanding, we only need high end GPU and high configuration device while training the network, once training is done, its dumps can be stored and using that one can easily make predictions and it can run on device with very low configuration easily. This model can be used in developing countries and rural areas, or wherever scarcity of ophthalmologist is observed or at places with less resources Other use of this tool can be as preliminary diagnosis tool for detecting diabetic retinopathy. This will help in earlier detection and faster treatment of diabetic retinopathy as it can only be treated in earlier stage. Our model shows that, to detect diabetic retinopathy in earlier stage becomes quite easy using this computer dependent tool.

Future Works, this network was trained on images of resolution $512 \times 512$ because of lack of hardware, but one can test it using higher resolution images. Another future enhancement can be training the network with unsupervised learning instead of pseudo-classification technique used in this model.

\section{References}

[1] L. Guariguata, D. Whiting, I. Hambleton, J. Beagley, U. Linnenkamp, and J. Shaw, "Global estimates of diabetes prevalence for 2013 and projections for 2035", Diabetes Research and Clinic Practice, Vol.103, No.2, pp.137-149, 2014.

[2] N. Patton, T. Aslam., T. MacGillivray, "Retinal image analysis: concepts, applications and potential", Progress in Retinal and Eye Research, pp.99-127, 2006.

[3] G. Bresnick, D. Mukamel, J. Dickinson, D. Cole, "A screening approach to the surveillance of patients with diabetes for the presence of visionthreatening retinopathy", Ophthalmology, Vol.107, No.1, pp.19-24, 2000.

[4] S. Philip, A. Fleming, K. Goatman, "The efficacy of automated 'disease/no disease' grading for diabetic retinopathy in a systematic screening programme", British Journal of Ophthalmology, Vol.91, No.11, pp.1512-1517, 2007.

[5] M. Abràmoff, M. Niemeijer, M. Suttorp-Schulten, M. Viergever, S. Russell, B. Ginneken,
"Evaluation of a system for automatic detection of diabetic retinopathy from color fundus photographs in a large population of patients with diabetes", Diabetes Care, Vol.31, No.2, pp.193198, 2008.

[6] B. Harangi, I. Lazar, A. Hajdu, "Automatic exudate detection using active contour model and region wise classification", In: Proc. of IEEE Conf. on Engineering in Medicine and Biology Society, pp.5951-5954, 2012.

[7] G. Hinton and R. Salakhutdinov, "Reducing the dimensionality of data with neural networks", Science, AAAS, Vol.313, No.5786, pp.504-507, 2006.

[8] L. Deng and D. Yu, "Deep learning: Methods and applications", Foundations and Trends ${ }^{\circledR}$ in Signal Processing, Vol.7, No.3-4, pp.197-387, 2014.

[9] G. Hinton et al., "Deep neural networks for acoustic modeling in speech recognition: The shared views of four research groups", IEEE Signal Processing Magazine, Vol.29, No.6, pp.82-97, 2012.

[10] L. Wan, M. Zeiler, S. Zhang, Y. LeCun, and R. Fergus, "Regularization of neural networks using DropConnect", In: Proc of the 30th International Conf. on Machine Learning (ICML-13), pp. 1058-1066, 2013.

[11] Y. Bengio, G. Mesnil, Y. Dauphin, and S. Rifai, "Better mixing via deep representations", In: Proc of 30th International Conf. on Machine Learning, pp.552-560, 2013.

[12] Y. Bengio, A. Courville, and P. Vincent, "Representation learning: A review and new perspectives", Pattern Analysis and Machine Intelligence, Vol.35, No.8, pp.1798-1828, 2013.

[13] T. Walter, "Detection of Retinal Pathologies from Images Color of the Eye", Ecole des Mines de Paris, Centre de Morphologie Mathématique, 2000.

[14] M. Cree, J. Olson, K. McHardy, P. Sharp, J. Forrester, "The preprocessing of retinal images for the detection of fluorescein leakage", Physics in Medicine and Biology, Vol.44, No.1, pp.293308, 1999.

[15] E. Peli, T. Peli, "Restoration of retinal images obtained through cataracts", Medical Imaging, Vol.8, No.4, pp.401-406, 1989.

[16] W. Hsu, P.M.D.S. Pallawa, M. Li Lee, K.-G. A Eong., "The role of domain knowledge in the detection of retinal hard exudates", In: Proc. of the IEEE Conf. on Computer Vision and Pattern Recognition, Vol. 2, pp. 246-251, 2001.

[17] T. Walter, J.-C. Klein, P. Massin, A. Erginay, “A contribution of image processing to the diagnosis 
of diabetic retinopathy - detection of exudates in color fundus images of the human retina", Medical Imaging, Vol.21, No.10, pp.1236-1243, 2002.

[18] X. Zhang, O. Chutatape.: "Detection and classification of bright lesions in colour fundus images", In: Proc of IEEE International Conf. on Image Processing, Vol.1, pp. 139-142, 2004.

[19] H. Wang, W. Hsu, K.G. Lee, M.L. Goh., "An effective approach to detect lesions in color retinal images", In: Proc. of the IEEE Conf. on Computer Vision and Pattern Recognition, pp.181-186, 2000.

[20] R.A. Welikalaa, J. Dehmeshki, A. Hoppe, V. Tahb, S. Mannc, T.H. Williamsonc, S.A. Barman, "Automated detection of proliferative diabetic retinopathy using a modified line operator and dual classification”, Computer Methods and Programs in Biomedicine, Vol.114, pp.247-261, 2014.

[21] X. Zhang, G. Thibault, E. Decencière, B. Marcotegui, B. Laÿ, R. Danno, G. Cazuguel, G. Quellec, M. Lamard, P. Massin, A. Chabouis, Z. Victor, A. Erginay, "Exudate Detection in Color Retinal Images for Mass Screening of Diabetic Retinopathy", Medical Image Analysis, 2014.

[22] M. Usman Akram, S. Khalid, A. Shoab-Khan, "Identification and classification of microaneurysms for early detection of diabetic retinopathy", Pattern Recognition, Vol.46, No.1, 2013.

[23] O. Faust, U.R. Acharya, E.Y. Ng, K.H. Ng, J.S. Suri, "Algorithms for the automated detection of diabetic retinopathy using digital fundus images: a review", Journal of Medical Systems, Vol.36, pp. 145-157, 2012.

[24] A. Tariq, M.U. Akram, A. Shaukat, S.A. Khan, "Automated detection and grading of diabetic maculopathy in digital retinal images", Journal of Digital. Imaging, Vol.26, pp.803-812, 2013.

[25] A.D. Fleming, S. Philip, K.A. Goatman, J.A. Olson, P.F. Sharp, "Automated microaneurysm detection using local contrast normalization and local vessel detection", Medical Imaging, Vol.25, No.9, pp.1223-1232, 2006. 